\title{
Late Onset Chemo/Radiation Induced Tracheoesophageal Fistula in Squamous Cell Cancer of the Lung
}

\author{
Ashraf Abugroun ${ }^{\mathrm{a}, \mathrm{b}}$, Fatima Ahmed ${ }^{\mathrm{a}}$, Namrata Singh ${ }^{\mathrm{a}}$, Mojtaba Nadiri ${ }^{\mathrm{a}}$
}

\begin{abstract}
We report a 72-year-old male with past medical history of stage IV squamous lung cancer treated with radiation therapy and chemotherapy with gemcitabine/carboplatin. One year later, he presented with hemoptysis treated with another session of palliative radiotherapy. Two weeks later, patient developed aspiration pneumonia and further workup showed trachea-esophageal fistula documented by barium swallow. This case shows a rare complication of chemotherapy and radiation therapy on the setting of primary lung cancer.
\end{abstract}

Keywords: Tracheoesophageal fistula; Squamous cells cancer; Radiotherapy; Chemotherapy; Gemcitabine/carboplatin

\section{Introduction}

A tracheoesophageal fistula (TEF) is an abnormal passage between the esophagus and the trachea that can be either congenital (incidence of one case in 2,000 - 4,000 live births) [1] or in rare cases acquired. Common causes for acquired TEF in adults include trauma, prolonged endotracheal intubation [2], and malignancy. There are sparse data on incidence of acquired malignant TEF as it is very rare. In few studies, incidence of TEF following primary esophageal cancer and lung cancer was reported at $4.5 \%$ and $0.3 \%$, respectively [3]. Chemotherapy and radiotherapy are associated with a relatively high incidence of TEF formation in lung cancer [4]. In the setting of previous radiation therapy, various anti-angiogenic agents were found to increase risk for fistula formation [5]. We here report a case of a 72-year-old male who developed a TEF upon re- irradiation of squamous cell cancer 1 year after treatment with gemcitabine/carboplatin. Patient had palliative stent placement to prevent further risk of aspiration pneumonia. This case highlights the role of radiotherapy and chemo-

Manuscript submitted October 20, 2017, accepted October 30, 2017

${ }^{a}$ Advocate Illinois Masonic Medical Center, Chicago, IL, USA

bCorresponding Author: Ashraf Abugroun, Advocate Illinois Masonic Medical Center, 836 W Wellington Ave., Chicago, IL 60657, USA.

Email: ashraf.abugroun@advocatehealth.com therapy on development of TEF on the setting of lung cancer.

\section{Case Report}

A 72-year-old male with past medical history of stage IV squamous lung cancer was brought to the hospital with complaints of anorexia, shortness of breath, and increased hemoptysis for preceding 4 weeks. His lung cancer was diagnosed 8 years earlier, when he presented with T4N2M0 non-small cell lung cancer treated with combined modality therapy consisting of chemotherapy and radiation to a dose of $6,480 \mathrm{cGy}$ in $180 \mathrm{cGy}$ fractions which led to a 7-year progression-free interval. One year earlier, he had recurrence of the cancer. Patient was not candidate for further radiation therapy. He received palliative chemotherapy with gemcitabine and carboplatin and after four cycles was placed on a chemotherapy holiday. Patient condition had been stable till 1 month prior to current admission when he developed progressive hemoptysis, right-sided chest pain and fatigue. On admission, chest X-ray (CXR) showed new worsened reticular markings along left lung base suggestive of infectious process. CT of chest showed findings consistent with interval progression of malignancy with an area of consolidation along the medial right lower lung (Fig. 1). Sputum culture showed mixed bacterial growth. Patient received short course of antibiotics followed by 10 fraction course of palliative radiation therapy. Immediately after completing his eighth cycle of radiotherapy, he developed progressive coughing and difficulty swallowing, together with shortness of breath, fever and confusion. He was readmitted, and CXR

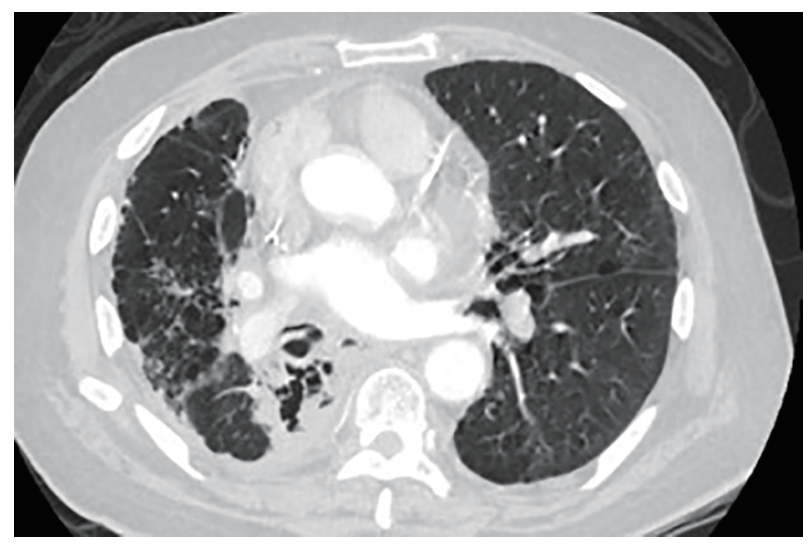

Figure 1. CT scan of the lung shows left apical necrotizing mass. 


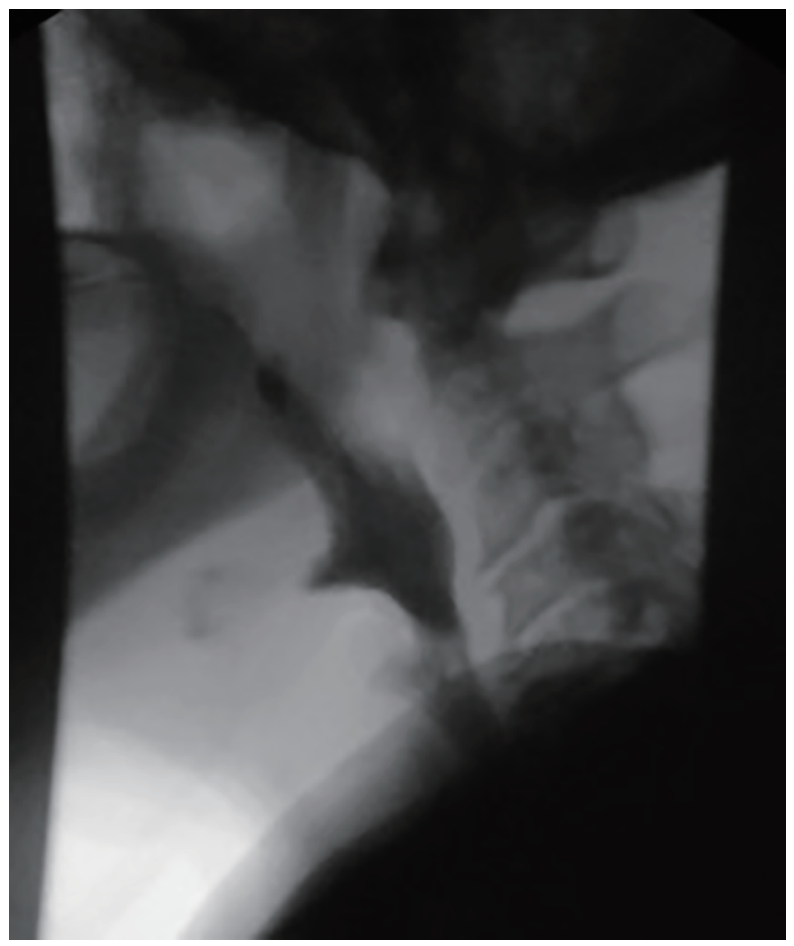

Figure 2. Video swallow evaluation shows a linear exclusion of contrast from mid esophagus into the tracheobronchial tree consistent with tracheobronchial fistula.

showed increased interstitial prominence at the bilateral lung bases. Laboratory workup revealed signs of infection with a WBC of $21.2 \times 10^{9} / \mathrm{L}$. Procalcitonin was $0.12 \mathrm{ng} / \mathrm{mL}$. He was diagnosed with hospital acquired pneumonia and received broad antibiotics coverage. His sputum culture revealed polymicrobial growth without organism identified. Due to concern of progressive coughing and difficulty swallowing a video, swallow evaluation was done. Surprisingly, it showed a TEF (Fig. 2) in the middle third of the esophagus. Patient underwent upper endoscopy and palliative esophageal stent placement (Fig. 3). Following successful stent placement, patient tolerated diet without complications. One week later, he was sent to hospice for further comfort measures.

\section{Discussion}

Acquired TEF is a known complication of lung and esophageal cancer. It is commonly reported as a complication of radiation therapy and on rare instances following chemotherapy [6]. TEF formed in the setting of malignancy signifies poor prognosis. Patients present mainly with intractable cough and repeated respiratory infections. Most patients die within a month of its development and the most common cause of death is recurrent aspiration pneumonitis and development of lung abscesses [4].

Most published data on acquired TEF in cancer setting are derived from case reports. Among patients treated for small cell lung cancer, TEF was reported in association with various chemotherapeutic agents including irinotecan, carboplatin, and bevacizumab. The mechanism for TEF formation in association with these medications is not fully understood. Radiation and chronic inflammation are important risk factors for fistula formation. Risk for fistula formation increases further with the use of chemotherapeutic agents that targets the VEGF pathway including sunitinib and bevacizumab as they can cause delayed healing secondary to impaired angiogenesis [5]. Interestingly, TEF following use of bevacizumab was reported in the absence of neither radiation nor direct involvement of the esophagus $[7,8]$. Such observations add to the complexity of understanding the mechanism of fistula formation in these settings.

The combination of carboplatin and gemcitabine is an effective regimen for the treatment of advanced squamous cell carcinoma of the lung. Carboplatin binds to DNA causing inhibition of its replication and transcription and inducing cell death. Gemcitabine is a pyrimidine analog that inhibits the enzyme ribonucleotide reductase, and thus inhibits DNA synthesis and cell growth. Both medications have high tolerability and act synergistically to suppress cell proliferation leading to cell death. Dose-limiting thrombocytopenia is the most common toxicity and rarely requires therapeutic intervention [911]. Development of TEF following the use of this medication was not reported. In this patient, the TEF developed 2 weeks

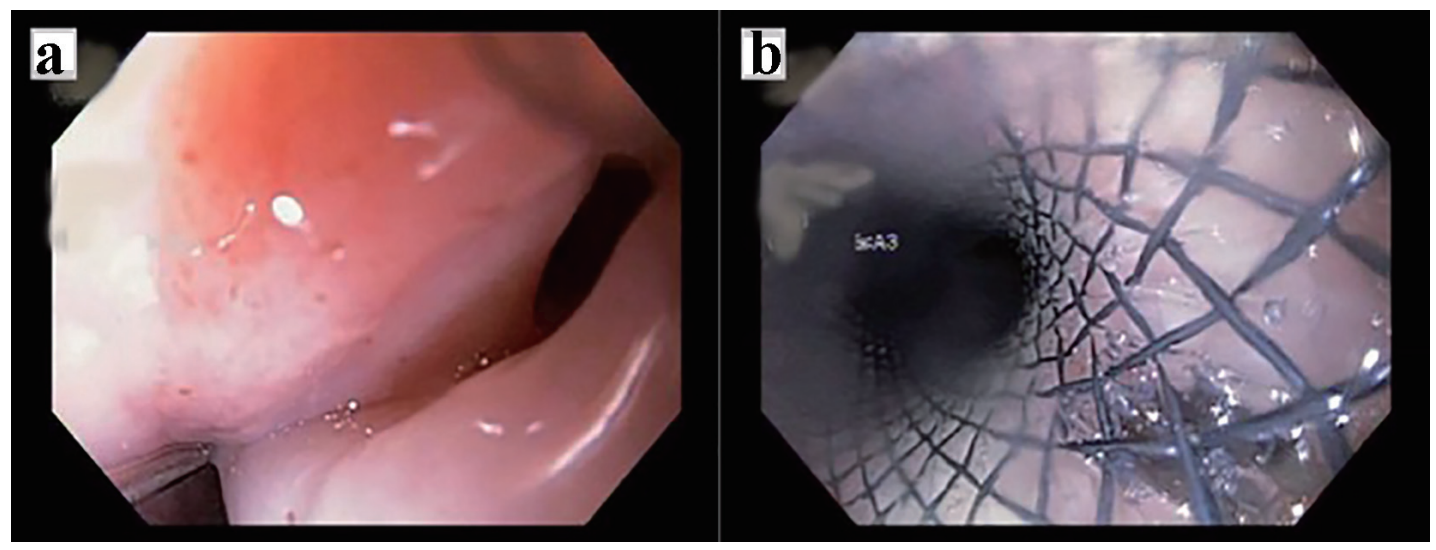

Figure 3. (A) Fistula in the middle third of the esophagus at about $25 \mathrm{~cm}$. (B) Prosthesis placed from approximately $20-30 \mathrm{~cm}$. Partially covered esophageal stent. 
following re-irradiation, so such short interval between development of the TEF and the radiotherapy points towards other contributing factors including local progression of the tumor and prior use of chemotherapy.

Treatment approach of TEF is usually individualized, based on the characteristics of the fistula and the patient's overall condition. Available options include surgical intervention, endovascular repair and conservative approach allowing spontaneous resolution with chemo-radiotherapy $[6,12,13]$.

\section{Conclusion}

TEF is a rare, often fatal complication of thoracic cancers and their treatments. It commonly presents with aspiration pneumonia and difficulty swallowing and heralds rapid decline in overall condition. Re-irradiation carries significant risk for esophageal fistula formation. The role of chemotherapeutic agents in pathogenesis of TEF formation is still not fully understood. Further studies are needed to study the role of nonantiangiogenic agents in TEF formation.

\section{References}

1. Tracheoesophageal fistula: background, pathophysiology, etiology. 2017 Jul 10 [cited 2017 Oct 28]; Available from: https://emedicine.medscape.com/article/186735overview.

2. Kaur D, Anand S, Sharma P, Kumar A. Early presentation of postintubation tracheoesophageal fistula: Perioperative anesthetic management. J Anaesthesiol Clin Pharmacol. 2012;28(1):114-116.

3. Burt M, Diehl W, Martini N, Bains MS, Ginsberg RJ, McCormack PM, Rusch VW. Malignant esophagorespiratory fistula: management options and survival. Ann Thorac Surg. 1991;52(6):1222-1228; discussion 1228-1229.

4. Duranceau A, Jamieson GG. Malignant tracheoesopha- geal fistula. Ann Thorac Surg. 1984;37(4):346-354.

5. Goodgame B, Veeramachaneni N, Patterson A, Govindan R. Tracheo-esophageal fistula with bevacizumab after mediastinal radiation. J Thorac Oncol. 2008;3(9):10801081.

6. Perry RR, Rosenberg RK, Pass HI. Tracheoesophageal fistula in the patient with lymphoma: case report and review of the literature. Surgery. 1989;105(6):770-777.

7. Spigel DR, Hainsworth JD, Yardley DA, Raefsky E, Patton J, Peacock N, Farley C, et al. Tracheoesophageal fistula formation in patients with lung cancer treated with chemoradiation and bevacizumab. J Clin Oncol. 2010;28(1):43-48.

8. Schreiber J, Waldburg N. Bronchoesophageal fistula and fatal hemoptysis after bevacizumab-containing chemotherapy without radiation in lung cancer. J Clin Oncol. 2012;30(32):e324.

9. Sousa GF de, Wlodarczyk SR, Monteiro G, Sousa GF de, Wlodarczyk SR, Monteiro G. Carboplatin: molecular mechanisms of action associated with chemoresistance. Braz J Pharm Sci. 2014;50(4):693-701.

10. Ramalingam S, Belani CP. Carboplatin/gemcitabine combination in advanced NSCLC. Oncology (Williston Park). 2004;18(8 Suppl 5):21-26.

11. Tassinari D, Fochessati F, Arcangeli V, Sartori S, Agostini V, Fantini M, Genestreti G, et al. Carboplatin and gemcitabine in the palliative treatment of stage IV non-small cell lung cancer: definitive results of a phase II trial. Tumori. 2004;90(1):54-59.

12. Nakamura M, Maruyama K, Furukawa J, Maruyama N, Shingai T, Katsumoto Y, Nakaguchi K, et al. [A patient with esophageal cancer with subcutaneal abscess and esophago-tracheal fistula who survived more than 2 years following treatment by drainage and chemoradiation therapy]. Gan To Kagaku Ryoho. 2001;28(11):1662-1665.

13. Rodriguez AN, Diaz-Jimenez JP. Malignant respiratorydigestive fistulas. Curr Opin Pulm Med. 2010;16(4):329333. 